\title{
An Efficient Approach to Multivariate Nakagami-m Distribution Using Green's Matrix Approximation
}

\author{
George K. Karagiannidis, Member, IEEE, Dimitris A. Zogas, Student Member, IEEE, and Stavros A. Kotsopoulos
}

\begin{abstract}
In this letter, an efficient approach for the evaluation of the Nakagami- $m$ multivariate probability density function (pdf) and cumulative distribution function (cdf) with arbitrary correlation is presented. Approximating the correlation matrix with a Green's matrix, useful closed formulas for the joint Nakagami- $m$ pdf and cdf, are derived. The proposed approach is a significant theoretical tool that can be efficiently used in the performance analysis of wireless communications systems operating over correlative Nakagami- $m$ fading channels.
\end{abstract}

Index Terms-Correlated fading, diversity systems, Nakagami- $m$ fading, Rayleigh fading, selection combining.

\section{INTRODUCTION}

D URING THE last years, there has been a continuing interest in modeling various wireless propagation channels with the generalized Nakagami- $m$ distribution, which provides optimum fits to collected data in indoor and outdoor mobile radio environments [1]. In wireless applications, as in diversity reception, which is used extensively to reduce the effect of fading on the system's performance in both fixed and mobile terminals, the usual assumption is that combined signals are independent of one another. However, this assumption is valid only if the diversity channels are sufficiently separated, which is not true for some applications, such as in wireless terminals with insufficient antenna spacing, equipped with space and polarization antenna diversity (mobile terminal, indoor base-station, etc). In such cases, the fading among the channels is correlative, resulting in a degradation of the diversity gain obtained [2]-[4]. Multivariate (correlated) Nakagami- $m$ and Rayleigh distributions analysis is an important tool in the performance investigation of the above-mentioned correlative fading applications, with space or frequency diversity and in multichannel reception. Moreover, it can be used to find the transition probabilities in first- (or higher) order Markov chain that can model the Nakagami- $m$ fading process [5], although Tan and Beaulieu in [6] refute the suitability of the Markovian assumption to the Nakagami- $m$ fading process. The usefulness of a functional form for the trivariate Nakagami- $m$ or Rayleigh probability density function (pdf) and a summary of works about multivariate Rayleigh densities were also referred in [7].

Manuscript received March 25, 2002; revised September 12, 2002; accepted September 17, 2002. The editor coordinating the review of this paper and approving it for publication is R. Murch.

G. K. Karagiannidis is with the Institute for Space Applications and Remote Sensing, National Observatory of Athens, 15236 Athens, Greece (e-mail: gkarag@space.noa.gr).

D. A. Zogas and S. A. Kotsopoulos are with the Electrical and Computer Engineering Department, University of Patras, 26442 Patras, Greece (e-mail: zogas@space.noa.gr; kotsop@ee.upatras.gr).

Digital Object Identifier 10.1109/TWC.2003.816792
From a literature review, there are few approaches to the Nakagami- $m$ multivariate analysis. Nakagami in [1] defined the bivariate- $m$ pdf. Later, Nakagami and Okui in [8] reported that the multivariate- $m$ pdf with exponential correlation has a closed easy maniputable form. Tan and Beaulieu in [9] presented an infinite series representation for the bivariate Nakagami- $m$ cumulative distribution function (cdf), while Simon and Alouini in [10] derived an expression for the bivariate Rayleigh cdf in the form of a single integral with finite limits and an integrand composed of elementary functions. Recently, the authors in [11] proposed a useful formulation for the multivariate $m$-distribution with exponential correlation. However, to our knowledge, an approach for the evaluation of the arbitrary correlated $n$-variate $(n>2)$ Nakagami- $m$ pdf and cdf, does not exist in the literature.

In this letter, capitalizing on the results for the generalized Rayleigh distribution in [12] and approximating the correlation matrix with a Green's matrix [13], a useful approach for the evaluation of the multivariate Nakagami- $m$ pdf and cdf, is presented. The proposed closed formulas can be efficiently used in the performance analysis of wireless applications, such as in space diversity systems (selection and switched diversity) or in Markov chain modeling of the Nakagami- $m$ fading channel.

\section{Multivariate NAKAGAMI- $m$ PDF AND CDF With ARBITRARY CORRELATION}

If $r$ is a Nakagami- $m$ variable, then its corresponding pdf is described by [1]

$$
f(r)=\frac{2 r^{2 m-1}}{\Gamma(m) \Omega^{m}} \exp \left(-\frac{r^{2}}{\Omega}\right), \quad r \geq 0
$$

with $\Gamma(\cdot)$ being the Gamma function, $\Omega=\overline{r^{2}} / m$, with $\overline{r^{2}}$ being the average signal power, and $m$ is the inverse normalized variance of $r^{2}$ which must satisfy $m \geq 1 / 2$, describing the fading severity. Moreover, it is well known that $r$ can be considered as the square root of the sum of squares of $m$ independent Rayleigh or $2 m$ independent Gaussian variates [1]. Let $\mathbf{Y}_{1}, \mathbf{Y}_{2}, \ldots, \mathbf{Y}_{2 m}$ be $n$-dimensional column vectors, which are independent and normally distributed with means zero and correlation matrix $\boldsymbol{\Sigma}$ given (without loss of generality) by $\boldsymbol{\Sigma}_{i, j} \equiv 1$ for $i=j$ and $\boldsymbol{\Sigma}_{i, j} \equiv \rho_{i, j}$ for $i \neq j$, with $0 \leq \rho_{i, j}<1$ and the inverse of $\boldsymbol{\Sigma}$ being tridiagonal. It is considered here that $\rho_{i, j}$ is the power correlation, which is the square of the correlation coefficient of the underlying Gaussian processes. Moreover, without loss of generality and for simplification purposes of the matrix $\Sigma$, it is assumed into the following that $\Omega_{i}=2 \sigma_{i}^{2}$, with $\sigma_{i}^{2}=1$ being the variance.

Let $\mathbf{X}_{1}, \mathbf{X}_{2}, \ldots, \mathbf{X}_{n}$ be $2 m$ dimensional column vectors, with $\mathbf{X}_{k}$ composed of the $k$ th components of the $\mathbf{Y}_{i}$ and 
$r_{1}=\left|\mathbf{X}_{1}\right|, r_{2}=\left|\mathbf{X}_{2}\right|, \ldots, r_{n}=\left|\mathbf{X}_{n}\right|$, where $\left|\mathbf{X}_{i}\right|$ means the norm of $\mathbf{X}_{i}$. Then, $r_{1}, r_{2}, \ldots, r_{n}$ are Nakagami- $m$ variables and following the same procedure as in the proof of Theorem I in [12], their joint pdf can be extracted as shown in (2), at the bottom of the page, with $\mathbf{W}$ being the inverse of $\boldsymbol{\Sigma}$, i.e., $\mathbf{W}=\boldsymbol{\Sigma}^{-1}$ with elements $p_{i, j}, 1 \leq i, j \leq n$, and $I_{\nu}(\cdot)$ is the first kind and $\nu$ th order modified Bessel function. Although the parameter $m$ in (2) seems to be restricted as a positive half-integer or integer, it can be any positive number not less than 0.5 , since (2) satisfies the necessary and sufficient conditions to be a joint distribution function, as it is also referred in [1] for the bivariate case. Setting $n=2$ in (2), the well-known bivariate Nakagami- $m$ pdf is derived [1], since for $m=1$ the $n$-variate Rayleigh pdf is resulted from (2) as

$$
\begin{aligned}
f\left(r_{1}, r_{2}, \ldots, r_{n}\right) & \\
= & |\mathbf{W}| r_{n} e^{-p_{n, n} r_{n}^{2} / 2} \\
& \cdot \prod_{k=1}^{n-1}\left[r_{k} e^{\left(-p_{k, k} r_{k}^{2} / 2\right)} I_{0}\left(\left|p_{k, k+1}\right| r_{k} r_{k+1}\right)\right] .
\end{aligned}
$$

The $n$-variate Nakagami- $m$ cdf is, by definition

$$
\begin{aligned}
& F\left(R_{1}, R_{2}, \ldots, R_{n}\right) \\
& \quad=\int_{0}^{R_{1}} \int_{0}^{R_{2}} \ldots \int_{0}^{R_{n}} f\left(r_{1}, r_{2}, \ldots, r_{n}\right) d r_{1} d r_{2} \ldots d r_{n}
\end{aligned}
$$

and following a similar procedure as in [9], resulting in (5), at the bottom of the page, with $\gamma(\cdot)$ being the incomplete Gamma function. The nested infinite series in (5) converges quickly for any values of $R_{1}, R_{2}, \ldots, R_{n}$ and any correlation matrix $\boldsymbol{\Sigma}$ (see Section V). For $n=2$, the bivariate Nakagami- $m$ cdf is derived as in [9], since for $m=1$ the $n$-variate Rayleigh cdf is given by (6), at the bottom of the page.

An upper bound for the error resulting after the truncation of the nested infinite series in (5) can be derived following a similar procedure as in [11].

\section{Selection COMBINER (SC) OUTPUT PDF AND CDF}

Equation (5), which describes the joint Nakagami- $m$ cdf, can be used to evaluate directly the performance of an SC, finding

$$
f\left(r_{1}, r_{2}, \ldots, r_{n}\right)=\frac{|\mathbf{W}|^{m} r_{1}^{m-1} r_{n}^{m} e^{-p_{n, n} r_{n}^{2} / 2}}{2^{m-1} \Gamma(m)} \prod_{k=1}^{n-1}\left[\left|p_{k, k+1}\right|^{-(m-1)} r_{k} e^{-p_{k, k} r_{k}^{2} / 2} I_{m-1}\left(\left|p_{k, k+1}\right| r_{k} r_{k+1}\right)\right]
$$

$$
\begin{aligned}
F\left(R_{1}, R_{2}, \ldots, R_{n}\right)= & \frac{|\mathbf{W}|^{m}}{\Gamma(m)} \times \sum_{i_{1}=0}^{\infty} \sum_{i_{2}=0}^{\infty} \ldots \sum_{i_{n-1}=0}^{\infty} \frac{\left|p_{1,2}\right|^{2 i_{1}}\left|p_{2,3}\right|^{2 i_{2}} \ldots\left|p_{n-1, n}\right|^{2 i_{n-1}}}{p_{1,1}^{i_{1}+m} p_{2,2}^{i_{1}+i_{2}+m} \ldots p_{n-1, n-1}^{i_{n-2}+i_{n-1}+m} p_{n, n}^{i_{n-1}+m}} \\
& \times\left(\prod_{j=1}^{n-1} \frac{1}{i_{j} ! \Gamma\left(i_{j}+m\right)}\right) \gamma\left(i_{1}+m, \frac{1}{2} p_{1,1} R_{1}^{2}\right) \\
& \times \gamma\left(i_{1}+i_{2}+m, \frac{1}{2} p_{2,2} R_{2}^{2}\right) \\
& \times \cdots \gamma\left(i_{n-2}+i_{n-1}+m, \frac{1}{2} p_{n-1, n-1} R_{n-1}^{2}\right) \\
& \times \gamma\left(i_{n-1}+m, \frac{1}{2} p_{n, n} R_{n}^{2}\right)
\end{aligned}
$$

$$
\begin{aligned}
F\left(R_{1}, R_{2}, \ldots, R_{n}\right)= & |\mathbf{W}| \times \sum_{i_{1}=0 i_{2}=0}^{\infty} \sum_{i_{n-1}=0}^{\infty} \frac{\left|p_{1,2}\right|^{2 i_{1}}\left|p_{2,3}\right|^{2 i_{2}} \ldots\left|p_{n-1, n}\right|^{2 i_{n-1}}}{p_{1,1}^{i_{1}+1} p_{2,2}^{i_{1}+i_{2}+1} \ldots p_{n-1, n-1}^{i_{n-2}+i_{n-1}+1} p_{n, n}^{i_{n}+1}} \\
& \times\left(\prod_{j=1}^{n-1} \frac{1}{i_{j} ! \Gamma\left(i_{j}+1\right)}\right) \gamma\left(i_{1}+1, \frac{1}{2} p_{1,1} R_{1}^{2}\right) \\
& \times \gamma\left(i_{1}+i_{2}+1, \frac{1}{2} p_{2,2} R_{2}^{2}\right) \\
& \times \cdots \gamma\left(i_{n-2}+i_{n-1}+1, \frac{1}{2} p_{n-1, n-1} R_{n-1}^{2}\right) \\
& \times \gamma\left(i_{n-1}+1, \frac{1}{2} p_{n, n} R_{n}^{2}\right)
\end{aligned}
$$


the one-dimensional cdf and pdf at the output. The SC output cdf can be used to evaluate the outage probability, while the SC output pdf for the evaluation of the average error probability and the average signal-to-noise ratio (SNR) [2]. As an example, let $R$ be the random variable that characterizes the output of a three-branches $\mathrm{SC}$ with inputs $R_{1}, R_{2}$, and $R_{3}$, i.e., $R=\max \left(R_{1}, R_{2}, R_{3}\right)$. Then, the cdf of $R$ is obtained evaluating the joint cdf of $R_{1}, R_{2}$, and $R_{3}$ using (5) for $n=3$ and equating the three arguments resulting in (7), at the bottom of the page. Differentiating (7), the pdf of $R$ can be written as

$$
\begin{aligned}
f_{\mathrm{SC}}(R)= & \frac{|\mathbf{W}|^{m}}{\Gamma(m)} \\
& \times \sum_{i_{1}=0}^{\infty} \sum_{i_{2}=0}^{\infty} \\
& \cdot \frac{\left[G_{1}+G_{2}+G_{3}\right]\left|p_{1,2}\right|^{2 i_{1}}\left|p_{2,3}\right|^{2 i_{2}}}{i_{1} ! i_{2} ! \Gamma\left(i_{1}+m\right) \Gamma\left(i_{2}+m\right) p_{1,1}^{i_{1}+m} p_{2,2}^{i_{1}+i_{2}+m} p_{3,3}^{i_{2}+m}}
\end{aligned}
$$

where

$$
\begin{aligned}
G_{1}= & \frac{1}{2^{i_{1}+m-1}} p_{1,1}^{i_{1}+m} R^{2\left(i_{1}+m\right)-1} e^{-p_{1,1} R^{2} / 2} \\
& \times \gamma\left(i_{1}+i_{2}+m, \frac{1}{2} p_{2,2} R^{2}\right) \gamma\left(i_{2}+m, \frac{1}{2} p_{3,3} R^{2}\right) \\
G_{2}= & \frac{1}{2^{i_{1}+i_{2}+m-1}} p_{2,2}^{i_{1}+i_{2}+m} R^{2\left(i_{1}+i_{2}+m\right)-1} e^{-p_{2,2} R^{2} / 2} \\
& \times \gamma\left(i_{1}+m, \frac{1}{2} p_{1,1} R^{2}\right) \gamma\left(i_{2}+m, \frac{1}{2} p_{3,3} R^{2}\right) \\
G_{3}= & \frac{1}{2^{i_{2}+m-1}} p_{3,3}^{i_{2}+m} R^{2\left(i_{2}+m\right)-1} e^{-p_{3,3} R^{2} / 2} \\
& \times \gamma\left(i_{1}+m, \frac{1}{2} p_{1,1} R^{2}\right) \gamma\left(i_{1}+i_{2}+m, \frac{1}{2} p_{2,2} R^{2}\right) .
\end{aligned}
$$

Following the same procedure, the cdf and the pdf of the output of an SC receiver with arbitrary number of branches, can be obtained.

\section{GREEN's MATRIX APPROXIMATION}

In the general case, the inverse of the correlation matrix $\mathbf{W}$ does not have the tridiagonal property and (2) does not apply. In this case, a useful form for the multivariate Nakagami- $m$ pdf with arbitrary correlation seems to be intractable. Hence, it is necessary to find such a way that the analysis of Section II being applicable for arbitrary correlation matrices. One answer to this problem, as proposed in this letter, is the approximation of $\boldsymbol{\Sigma}$ with another matrix $\mathbf{C}$ with elements the closest possible values to the entries of $\boldsymbol{\Sigma}$, in order $\mathbf{C}^{-1}$ to be tridiagonal. A symmetric, irreducible nonsingular matrix, is tridiagonal, if and only if its inverse is a Green's matrix [13]. Hence, in our case $\mathbf{C}$ must have the form

$$
\mathbf{C}=\left(\begin{array}{ccccc}
u_{1} v_{1} & u_{1} v_{2} & & \cdots & u_{1} v_{n} \\
u_{1} v_{2} & u_{2} v_{2} & & & u_{2} v_{n} \\
& & & & \vdots \\
\vdots & & & \ddots & \\
u_{1} v_{n} & u_{2} v_{n} & \cdots & & u_{n} v_{n}
\end{array}\right)
$$

with $u_{i}$ and $v_{i}, i=1, \ldots, n$, be two sequences of real numbers, and $u_{i} v_{i}=1$, due to the form of $\boldsymbol{\Sigma}$. Equating $\boldsymbol{\Sigma}$ with $\mathbf{C}$, a nonlinear system equation is produced, as shown in (10), at the bottom of the page.

This system can be solved for $v_{i}$ using well-known nonlinear methods as Levenberg-Marquardt, quasi-Newton, or conjugate gradient [15], available in most well-known mathematical software packages, as MATHEMATICA, MATLAB, MAPLE, etc. Since $u_{i}=1 / v_{i}, \mathbf{C}$ can be defined and the analysis of Section II can also be applied using matrix $\mathbf{C}$ as the new (approximated) correlation matrix.

\section{NUMERICAL-SIMULATION RESULTS AND DISCUSSION}

In this section, numerical examples and simulation results are provided, for several correlation models, well known from practical diversity systems [2], in order to provide the applicability and check the usefulness of the proposed analysis.

$$
\begin{aligned}
F_{\mathrm{SC}}(R)= & \frac{|\mathbf{W}|^{m}}{\Gamma(m)} \times \sum_{i_{1}=0}^{\infty} \sum_{i_{2}=0}^{\infty} \frac{\left|p_{1,2}\right|^{2 i_{1}}\left|p_{2,3}\right|^{2 i_{2}}}{i_{1} ! i_{2} ! \Gamma\left(i_{1}+m\right) \Gamma\left(i_{2}+m\right) p_{1,1}^{i_{1}+m} p_{2,2}^{i_{1}+i_{2}+m} p_{3,3}^{i_{2}+m}} \\
& \times \gamma\left(i_{1}+m, \frac{1}{2} p_{1,1} R^{2}\right) \gamma\left(i_{1}+i_{2}+m, \frac{1}{2} p_{2,2} R^{2}\right) \\
& \times \gamma\left(i_{2}+m, \frac{1}{2} p_{3,3} R^{2}\right)
\end{aligned}
$$

$$
\begin{array}{llll}
v_{2}=\boldsymbol{\Sigma}_{2,1} v_{1} & \\
v_{3}=\boldsymbol{\Sigma}_{3,1} v_{1} & v_{3}=\boldsymbol{\Sigma}_{3,2} v_{2} & \\
v_{4}=\boldsymbol{\Sigma}_{4,2} v_{2} & v_{4}=\boldsymbol{\Sigma}_{4,3} v_{3} & \\
\vdots & v_{5}=\boldsymbol{\Sigma}_{5,3} v_{3} & \ddots & \ddots \\
v_{n}=\boldsymbol{\Sigma}_{n, 1} v_{1} & v_{n}=\boldsymbol{\Sigma}_{n, 2} v_{2} & v_{n}=\boldsymbol{\Sigma}_{n, 3} v_{3} & \ldots \ldots
\end{array}
$$




\section{A. Exponential Model}

The correlation matrix of this model is defined by [3] as $\boldsymbol{\Sigma}_{i, j} \equiv 1$ for $i=j$ and $\boldsymbol{\Sigma}_{i, j} \equiv \rho^{|i-j|}$ for $i \neq j$ and corresponds to the scenario of multichannel reception from equispaced diversity antennas, in which the correlation between the pairs of combined signals decays as the spacing between the antennas increases [2, p. 324]. The exponential model was used by several researchers who deal with the performance analysis of space diversity techniques [2], and is close to the practical situation of linear arrays, though it requires the antennas to be placed unevenly [4]. It is well known that the inverse of the exponential correlation matrix, also called the Markov matrix [16], is tridiagonal. Hence, the multivariate Nakagami- $m$ pdf and cdf can be evaluated directly using (2) and (5), which after manipulations can be simplified as shown in (11), at the bottom of the page, with

$$
g_{1}=\left\{\begin{array}{l}
0, \quad \text { for } n=2 \\
\frac{\rho^{2}+1}{2\left(\rho^{2}-1\right)} \sum_{k=2}^{n-1} r_{k}^{2}, \quad \text { for } n>2
\end{array}\right\}
$$

The exponentially correlated multivariate Nakagami- $m$ distribution has been treated in detail in [11]. The corresponding multivariate cdf can be written as shown in (12), at the bottom of the page, with

$g_{2}=\left\{\begin{array}{l}1, \text { for } n=2 \\ \left(\rho^{2}+1\right)^{-\left[{ }^{i_{1}+2 i_{2}+\cdots+2 i_{n-2}+i_{n-1}+(n-2) m}\right]}, \text { for } n>2\end{array}\right\}$.

As referred above, the nested infinite series in (12) converges rapidly with a significant speedup factor compared to numerical integration techniques used to evaluate the multiple integral of (4). In Fig. 1, the number of required terms for each sum $I_{i}$ is depicted in order to obtain seven significant figure accuracy for the trivariate and the quatrivariate case, using (12). These terms are calculated empirically using MAPLE 7. For simplicity it is assumed here that $I_{1}=I_{2}=\cdots=I_{n-1}$. As it is shown in this figure, an increase to the correlation leads also to an increase of the required terms need to be summed in order to obtain a given accuracy. Furthermore, the number of the required terms depends strongly on the signals envelope and increases proportionally with it. However, this increase is smoother than the corresponding in the bivariate case [9]. It is also interesting to observe here that the nested infinite series representation itself converges and gives results for all values of $\rho$ between zero and one. Even for the extreme case of $\rho=0.99$, (12) also gives accurate results with $I_{1}=I_{2}=40$ and $I_{1}=I_{2}=52$ for $\{\mathrm{m}=$ $\left.2, R_{1}=R_{2}=R_{3}=1\right\}$ and $\left\{m=2, R_{1}=R_{2}=1, R_{3}=10\right\}$, correspondingly.

\section{B. Linearly Arbitrary Model}

This is a more general case than the exponential one with a correlation matrix $\boldsymbol{\Sigma}_{i, j} \equiv 1$ for $i=j$ and $\boldsymbol{\Sigma}_{i, j} \equiv \rho_{i, j}$ for $i \neq j$. This matrix has a Toeplitz structure. In this case, the inverse of $\boldsymbol{\Sigma}$ is not a tridiagonal matrix and a Green's matrix approximation is needed, following the procedure of Section IV. The correlation coefficients decrease as the distance between the elements of $\boldsymbol{\Sigma}$ increase. This kind of correlation corresponds to the practical situation of linear arrays, with the antennas not to be placed unevenly. An example of a correlation matrix from a linear array with three branches created by empirical curves was given in [4] as

$$
\boldsymbol{\Sigma}_{3 \_ \text {lin }}=\left[\begin{array}{lll}
1.000 & 0.795 & 0.605 \\
0.795 & 1.000 & 0.795 \\
0.605 & 0.795 & 1.000
\end{array}\right] .
$$

$$
\begin{aligned}
f_{\exp }\left(r_{1}, r_{2}, \ldots, r_{n}\right)= & \frac{1}{2^{m-1} \Gamma(m)} \times\left[\frac{1}{\left(1-\rho^{2}\right)^{n-1}}\right]^{m} r_{1}^{m-1} r_{n}^{m} e^{\left[\left(r_{1}^{2}+r_{n}^{2}\right) / 2\left(\rho^{2}-1\right)\right]+g_{1}} \\
& \times \prod_{k=1}^{n-1}\left[\left|\frac{\rho}{\rho^{2}-1}\right|^{-(m-1)} r_{k} I_{m-1}\left(\left|\frac{\rho}{\rho^{2}-1}\right| r_{k} r_{k+1}\right)\right]
\end{aligned}
$$

$$
\begin{aligned}
F_{\exp }\left(R_{1}, R_{2}, \ldots, R_{n}\right)= & \frac{\left(1-\rho^{2}\right)^{m}}{\Gamma(m)} \times \sum_{i_{1}=0}^{\infty} \sum_{i_{2}=0}^{\infty} \ldots \sum_{i_{n-1}=0}^{\infty} g_{2} \rho^{2 i_{1}+2 i_{2}+\cdots+2 i_{n-1}} \\
& \times\left(\prod_{j=1}^{n-1} \frac{1}{i_{j} ! \Gamma\left(i_{j}+m\right)}\right) \gamma\left(i_{1}+m, \frac{1}{2\left(1-\rho^{2}\right)} R_{1}^{2}\right) \\
& \times \gamma\left(i_{1}+i_{2}+m, \frac{1}{2}\left(\frac{\rho^{2}+1}{1-\rho^{2}}\right) R_{2}^{2}\right) \\
& \times \cdots \gamma\left(i_{n-2}+i_{n-1}+m, \frac{1}{2}\left(\frac{\rho^{2}+1}{1-\rho^{2}}\right) R_{n-1}^{2}\right) \\
& \times \gamma\left(i_{n-1}+m, \frac{1}{2\left(1-\rho^{2}\right)} R_{n}^{2}\right) .
\end{aligned}
$$




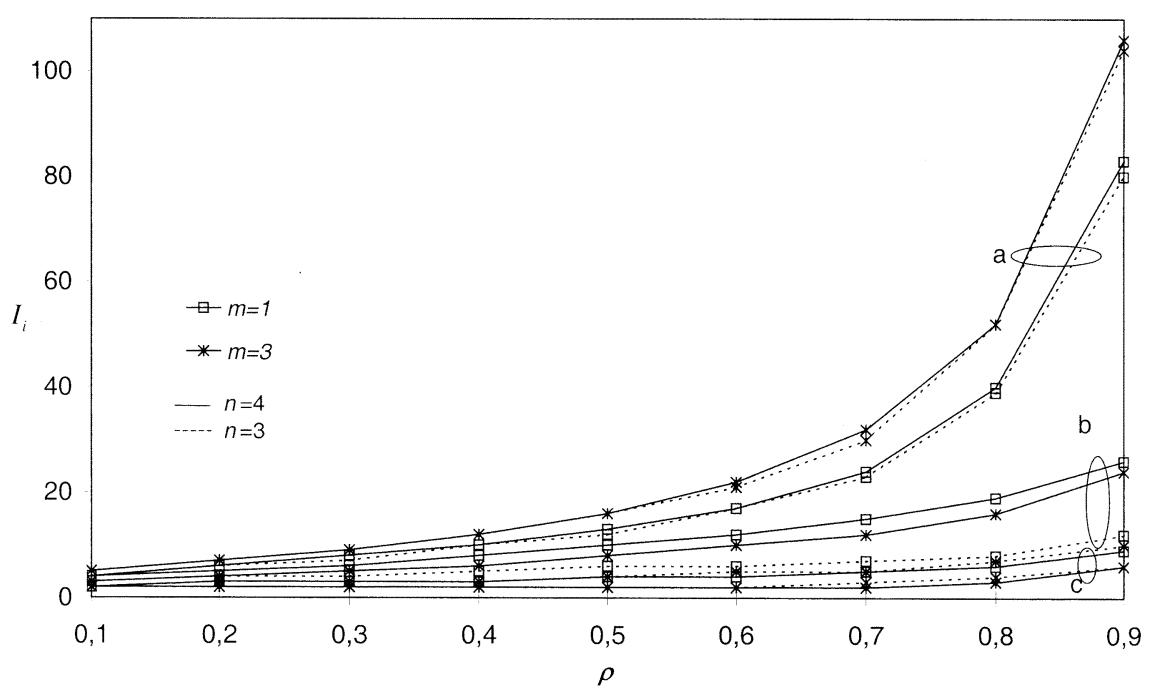

Fig. 1. Number of terms need to be summed for each sum of the infinite series of (12) versus the correlation coefficient $\rho$. (a) $\left[\left(R_{1}=R_{2}=R_{3}=R_{4}=1\right),\left(R_{1}=\right.\right.$ $\left.\left.R_{2}=R_{3}=1\right)\right]$. (b) [ $\left.\left(R_{1}=R_{2}=1, R_{3}=R_{4}=10\right),\left(R_{1}=R_{2}=1, R_{3}=10\right)\right]$. (c) [ $\left.\left(R_{1}=R_{2}=R_{3}=R_{4}=10\right),\left(R_{1}=R_{2}=R_{3}=10\right)\right]$.

In this case, $\boldsymbol{\Sigma}_{3 \_ \text {lin }}^{-1}$ is not tridiagonal and its corresponding Green's matrix can be found using the Levenberg-Marquardt method as

$$
\mathbf{C}_{3 \_ \text {lin }}=\left[\begin{array}{lll}
1.000 & 0.786 & 0.617 \\
0.786 & 1.000 & 0.786 \\
0.617 & 0.786 & 1.000
\end{array}\right] \text {. }
$$

Another example for a four-branches linear array is

$$
\boldsymbol{\Sigma}_{4 \_ \text {lin }}=\left[\begin{array}{cccc}
1.000 & 0.786 & 0.617 & 0.450 \\
0.786 & 1.000 & 0.750 & 0.620 \\
0.620 & 0.750 & 1.000 & 0.750 \\
0.450 & 0.62 & 0.750 & 1.000
\end{array}\right]
$$

which is approximated using the Levenberg-Marquardt method with the matrix

$$
\mathbf{C}_{4 \_ \text {lin }}=\left[\begin{array}{cccc}
1.000 & 0.700 & 0.538 & 0.377 \\
0.700 & 1.00 & 0.769 & 0.538 \\
0.538 & 0.769 & 1.000 & 0.700 \\
0.377 & 0.538 & 0.700 & 1.000
\end{array}\right]
$$

The above examples will be used below to check the accuracy of the proposed analysis.

\section{Constant Model}

The constant correlation model, discussed in [2] and [3], is related to the situation of $L$ identically distributed Nakagami- $m$ channels with constant correlation across all channels. In such a case, the correlation matrix is defined as $\boldsymbol{\Sigma}_{i, j} \equiv 1$ for $i=j$ and $\boldsymbol{\Sigma}_{i, j} \equiv \rho$ for $i \neq j$. In the context of antenna diversity, the spatial correlation is a function of the distance between the antennas and this model will then apply to equidistant antennas. This situation corresponds to size-limited scenarios with diversity reception from an array of three antennas placed on an equilateral triangle or from closely placed antennas on other than linear arrays. It must be mentioned here that one may expect that an antenna array with a totally symmetrical triangular configuration would have a constant correlation. But, this is not true since the signal incident angle also has an impact on the branch correlation, and it is never symmetrical to all three broadsides of a triangle [4]. In the case of constant correlation, the inverse of $\boldsymbol{\Sigma}$ is not a tridiagonal matrix and Green's matrix approximation is also needed. As an example of constant correlation in the case of antenna arrays with correlation matrices given by

$$
\begin{aligned}
\Sigma_{3 \text { _con }} & =\left[\begin{array}{lll}
1.000 & 0.600 & 0.600 \\
0.600 & 1.000 & 0.600 \\
0.600 & 0.600 & 1.000
\end{array}\right] \\
\text { and } \boldsymbol{\Sigma}_{4 \text { _con }} & =\left[\begin{array}{llll}
1.000 & 0.500 & 0.500 & 0.500 \\
0.500 & 1.000 & 0.500 & 0.500 \\
0.500 & 0.500 & 1.000 & 0.500 \\
0.500 & 0.500 & 0.500 & 1.000
\end{array}\right]
\end{aligned}
$$

the corresponding Green's matrices are obtained using the Levenberg-Marquardt method as

$$
\begin{aligned}
\mathbf{C}_{3 \_ \text {con }} & =\left[\begin{array}{lll}
1.000 & 0.699 & 0.473 \\
0.699 & 1.000 & 0.699 \\
0.473 & 0.699 & 1.000
\end{array}\right] \\
\text { and } \mathbf{C}_{4 \_ \text {con }}= & {\left[\begin{array}{llll}
1.000 & 0.641 & 0.434 & 0.278 \\
0.641 & 1.000 & 0.676 & 0.434 \\
0.434 & 0.676 & 1.000 & 0.641 \\
0.278 & 0.434 & 0.641 & 1.000
\end{array}\right] . }
\end{aligned}
$$

\section{Circular Model}

In the case of circular correlation [17], the correlation matrix is not only symmetric, but also has $n$th order symmetry, i.e.,

$$
\boldsymbol{\Sigma}_{\text {circ }}=\left(\begin{array}{ccccc}
1 & \rho_{1} & \rho_{2} & \cdots & \rho_{n-1} \\
\rho_{1} & 1 & \ddots & & \vdots \\
\rho_{2} & \ddots & \ddots & & \rho_{2} \\
\vdots & & & 1 & \rho_{1} \\
\rho_{n-1} & \cdots & \rho_{2} & \rho_{1} & 1
\end{array}\right)
$$

which implies that $\rho_{1}=\rho_{n-1}, \rho_{2}=\rho_{n-2}, \ldots$. This model may apply to antennas lying on a circle or four antennas placed on a square. It must be noted here that the applicability of this model 


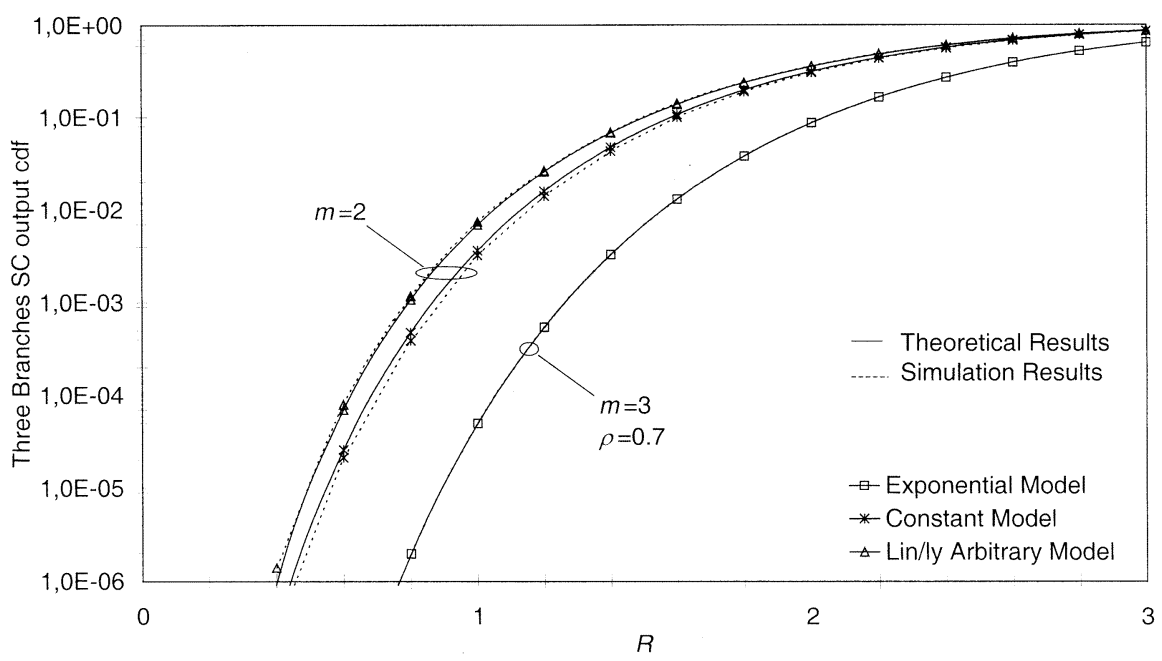

Fig. 2. Theoretical and simulation results for a three-branches SC output cdf.

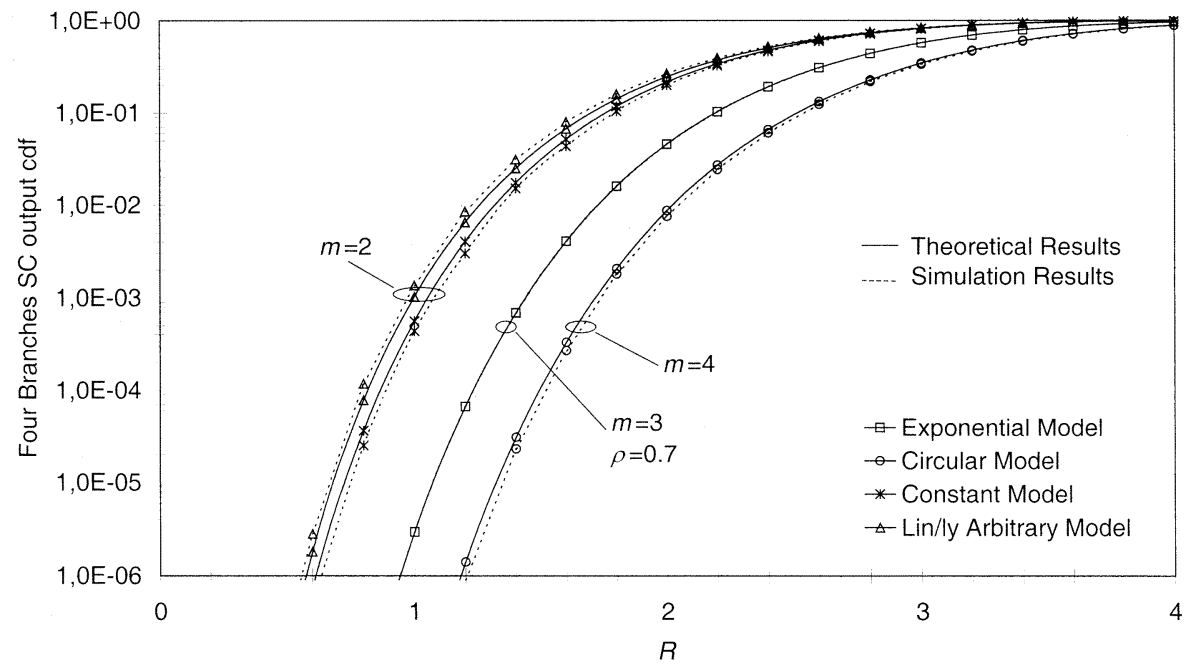

Fig. 3. Theoretical and simulation results for a four-branches SC output cdf.

in practical situations is questionable since the signal incident angle also has impact on the branch correlation and it is never symmetrical to all four edges of a circle or a polygon. As an example of circular model could be the case of four antennas placed on the edges of a square with correlation matrix

$$
\boldsymbol{\Sigma}_{4 \_ \text {circ }}=\left[\begin{array}{cccc}
1.000 & 0.700 & 0.500 & 0.700 \\
0.700 & 1.000 & 0.700 & 0.500 \\
0.500 & 0.700 & 1.000 & 0.700 \\
0.700 & 0.500 & 0.700 & 1.000
\end{array}\right]
$$

The inverse of this type of matrix is not tridiagonal and the closest Green's matrix approximation was obtained using the Levenerg-Marquardt method as

$$
\mathbf{C}_{4 \_ \text {circ }}=\left[\begin{array}{llll}
1.000 & 0.786 & 0.572 & 0.453 \\
0.786 & 1.000 & 0.732 & 0.576 \\
0.572 & 0.732 & 1.000 & 0.786 \\
0.453 & 0.576 & 0.786 & 1.000
\end{array}\right]
$$

\section{E. Results and Discussion}

In order to check the accuracy and the usefulness of the proposed Green's matrix approximation technique, simulation was performed for the arbitrary correlation case and the results were compared to the corresponding from the mathematical analysis, after the Green's matrix approximation. Using the examples presented above, the cdf at the output of three- and fourbranches SC are depicted in Figs. 2 and 3, correspondingly. The computer simulation was performed using the $\mathrm{C}^{++}$programming language. The algorithm that has been used is presented in [18]. For the generation of the correlated Nakagami- $m$ fading envelops, over a million samples were used. Some comments on the applicability of the analysis proposed in this letter to the above-described correlation models can be derived from Figs. 2 and 3. As was expected, (2) and (5) for the multivariate Nakagami- $m$ pdf and cdf can be applied directly in the case of the exponential correlation, which is used in the important practical case of linear antenna arrays. The simulation results were the same as the outcomes from the numerical analysis. Moreover, for the other correlation models (linearly arbitrary, constant, circular), the Green's matrix approximation gives accurate results 
and the analysis of Section II can be efficiently applied without a considerable effect. This is true, when performance evaluation techniques as the calculation of the outage probability are used.

\section{CONCLUSION}

In this letter, a useful approach to the joint $n$-variate Nakagami- $m$ pdf and cdf with arbitrary correlation is presented. After simulation, it was observed that the proposed approach could be efficiently applied in the performance analysis of diversity systems operating over arbitrary correlated Nakagami- $m$ or Rayleigh fading channel. Moreover, it can be used in other applications as in finding the transition probabilities in the first (or higher order) Markov chain modeling of the Nakagami- $m$ fading channel. ${ }^{1}$

\section{ACKNOWLEDGMENT}

The authors would like to thank N. C. Sagias for the validation of the mathematical analysis by simulations. They would also like to thank the anonymous reviewers and the Editor, R. Murch, for their remarks and suggestions that considerably improved the quality of this letter.

\section{REFERENCES}

[1] M. Nakagami, "The $m$-distribution - A general formula for intensity distribution of rapid fading," in Statistical Methods in Radio Wave Propagation, W. G. Hoffman, Ed. Oxford, U.K.: Pergamon, 1960, pp. 3-36.

[2] M. K. Simon and M.-S. Alouini, Digital Communication Over Fading Channels, 1st ed. New York: Wiley, 2000.

${ }^{1}$ Very recently, another useful work on the multivariate Rayleigh distribution was presented in [19].
[3] V. A. Aalo, "Performance of maximal - ratio diversity systems in a correlated Nakagami - Fading environment," IEEE Trans. Commun., vol. 43, pp. 2360-2369, Aug. 1995.

[4] Q. T. Zhang, "Maximal-ratio combining over Nakagami fading channels with an arbitrary branch covariance matrix," IEEE Trans. Veh. Technol., vol. 48, pp. 1141-1150, July 1999.

[5] H. S. Wang and P.-C. Chang, "On verifying the first order Marcovian assumption for a Rayleigh fading channel model," IEEE Trans. Veh. Technol., vol. 45, pp. 353-357, May 1996.

[6] C. C. Tan and N. C. Beaulieu, "On first-order Markov modeling for the Rayleigh fading channel," IEEE Trans. Commun., vol. 48, pp. 2032-2040, Dec. 1995.

[7] A. Abdi, "Comments on 'On verifying the first order Marcovian assumption for a Rayleigh fading channel model'," IEEE Trans. Veh. Technol., vol. 48, pp. 1739-1739, Sept. 1999.

[8] M. Nakagami and S. Okui, "The multivariable correlated distribution of type m," Nat. Convention Record IECE, vol. 13, p. 39, 1964.

[9] C. C. Tan and N. C. Beaulieu, "Infinite series representation of the bivariate Rayleigh and Nakagami- $m$ distributions," IEEE Trans. Commun., vol. 45, pp. 1159-1161, Oct. 1997.

[10] M. K. Simon and M.-S. Alouini, "A simple integral representation of the bivariate Rayleigh distribution," IEEE Commun. Lett., vol. 2, pp. 128-130, May 1998.

[11] G. K. Karagiannidis, D. A. Zogas, and S. A. Kotsopoulos, "On the multivariate Nakagami- $m$ distribution with exponential correlation," IEEE Trans. Commun., vol. 51, pp. 1240-1244, Aug. 2003.

[12] L. E. Blumenson and K. S. Miller, "Properties of generalized Rayleigh distributions," Annu. Math. Statist., vol. 34, pp. 903-910, 1963.

[13] R. Nabben, "On Green's matrices of trees," SIAM J. Matrix Anal. Appl., vol. 4, pp. 1014-1026, 2000.

[14] I. S. Gradshteyn and I. M. Ryzhik, Table of Integrals, Series, and Products, 5th ed. New York: Academic, 1994.

[15] G. H. Golub and C. F. Van Loan, Matrix Computations, 3rd ed. Baltimore, MD: The Johns Hopkins Univ. Press, 1996.

[16] D. R. Jensen, "A generalization of the multivariable rayleigh distribution," Sankhya: Series A, vol. 32, pp. 193-206, 1970.

[17] M.-S. Alouini, A. Abdi, and M. Kaveh, "Sum of gamma variates and performance of wireless communication systems over Nakagami-fading channels," IEEE Trans. Veh. Technol., vol. 50, pp. 1471-1480, Nov. 2001.

[18] Y.-C. Ko, "Analysis techniques for the performance evaluation of wireless communication systems and estimation of wireless channels," Ph.D. dissertation, Univ. Minesota, Minneapolis, MN, 2001.

[19] R. K. Mallik, "On multivariate Rayleigh and exponential distributions," IEEE Trans. Inform. Theory, vol. 49, pp. 1499-1515, June 2003. 